\title{
Factor VIII activity and bleeding risk during prophylaxis for severe hemophilia A: a population pharmacokinetic model
}

\author{
Andreas Tiede, ${ }^{1}$ Faraizah Abdul Karim, ${ }^{2}$ Victor Jiménez-Yuste, ${ }^{3}$ \\ Robert Klamroth, ${ }^{4}$ Sandra Lejniece, ${ }^{5}$ Takashi Suzuki, ${ }^{6}$ Andreas Groth ${ }^{7}$ and \\ Elena Santagostino 8

\begin{abstract}
${ }^{1}$ Hannover Medical School (MHH), Hematology, Hemostasis, Oncology and Stem Cell Transplantation, Hannover, Germany; ${ }^{2}$ Haemophilia Centre, National Blood Centre, Kuala Lumpur, Malaysia; ${ }^{3}$ Hospital Universitario La Paz, Autónoma University, Madrid, Spain; ${ }^{4}$ Haemophiliezentrum, Klinik für Innere Medizin, Vivantes Klinikum im and Hematology Clinic, Rîga, Latvia; ${ }^{6}$ Department of Laboratory Medicine, Tokyo Medical University, Tokyo, Japan; ${ }^{7}$ Novo Nordisk A/S, Søborg, Denmark and ${ }^{8}$ Angelo Bianchi Bonomi Hemophilia and Thrombosis Center, IRCCS Cà Granda Foundation, Maggiore Hospital Policlinic, Milan, Italy
\end{abstract} \\ Friedrichshain, Berlin, Germany; ${ }^{5}$ Rîga East Clinical University Hospital, Chemotherapy
}

Haematologica 2021

Volume 106(7):1902-1909

\section{ABSTRACT}

$\longrightarrow$ uring factor VIII prophylaxis for severe hemophilia A, bleeding risk increases with time when factor VIII activity is below $1 \%$. However, maintaining trough activity above $1 \%$ does not protect all patients from bleeding. The relationship between factor VIII activity during prophylaxis and bleeding risk has not been thoroughly studied. We investigated factor VIII activity and annualized bleeding rate for spontaneous bleeds during prophylaxis. A population pharmacokinetic model derived from three clinical trials was combined with dosing data and information on bleeding from patients' diaries. Each patient's time on prophylaxis was divided into five categories of predicted activity $(0-1 \%,>1-5 \%,>5-15 \%$, $>15-50 \%$, and $>50 \%$ ). Exposure time, mean factor VIII activity, and number of bleeds (from the patients' diaries) were calculated for each activity category, and annualized bleeding rates estimated using negative binomial regression and a parametric model. Relationships between these bleeding

\section{Correspondence:}

ANDREAS TIEDE

tiede.andreas@mh-hannover.de

Received: November 27, 2019.

Accepted: April 22, 2020.

Pre-published: April 23, 2020.

https://doi.org/10.3324/haematol.2019.241554

(C)2021 Ferrata Storti Foundation

Material published in Haematologica is covered by copyright. All rights are reserved to the Ferrata Storti Foundation. Use of published material is allowed under the following terms and conditions:

https://creativecommons.org/licenses/by-nc/4.0/legalcode. Copies of published material are allowed for personal or internal use. Sharing published material for non-commercial purposes is subject to the following conditions:

https://creativecommons.org/licenses/by-nc/4.0/legalcode, sect. 3. Reproducing and sharing published material for commercial purposes is not allowed without permission in writing from the publisher. rates and factor VIII activity were evaluated by trial phase (pivotal vs. extension) and age (adults/adolescents [ $\geq 12$ years] vs. children $[0-<12$ years]). In total ( $\mathrm{n}=187$ patients; 815 patient-years' exposure), factor VIII activity was predicted to be $>1 \%$ for $85.64 \%$ of the time. The annualized bleeding rate decreased as factor VIII activity increased in each trial phase and age group. However, for a given activity level, bleeding rate differed substantially by trial phase and age. This suggests that bleeding risk can change over time and is influenced by factors independent of factor VIII pharmacokinetics and trough levels. When making decisions regarding target trough levels and the prophylactic regimen, the patients' age, joint disease activity, and other bleeding risk determinants should be taken into consideration. Clinical trial registration numbers: NCT00840086; NCT01138501; NCT00984126

\section{Introduction}

Hemophilia is classified according to factor VIII (FVIII) plasma activity as "severe" ( $<1 \%$ of normal activity), "moderate" $(1-5 \%)$, or "mild" $(>5 \%-<40 \%),{ }^{1,2}$ Severe hemophilia is characterized by spontaneous, recurrent bleeds into joints and muscles which can lead to chronic arthropathy, muscular atrophy, and deformities. Converting the clinical phenotype of hemophilia from severe to moderate has been the rationale for prophylaxis. ${ }^{3}$ In patients with severe hemophilia A, prophylaxis with replacement FVIII can prevent bleeds and structural joint damage when initiated at a young age, ${ }^{4}$ and can decrease bleeding frequency, slow joint disease progression, and improve quality of life - even when initiated in adults with established joint damage. ${ }^{5}$ 
In severe hemophilia $A$, the annualized bleeding rate (ABR) during prophylaxis was shown to correlate with time spent with FVIII activity (FVIII:C) below 1\%, as predicted from the patient's individual FVIII pharmacokinetics (PK). ${ }^{6}$ However, actual time below $1 \%$ or any other targeted trough level also depends on the prescribed prophylaxis regimen and the patient's adherence to it. The risk of spontaneous bleeds during periods with FVIII:C $\geq 1 \%$ in subjects on prophylaxis is unknown, although an epidemiological study in individuals with mild/moderate hemophilia suggested that maintaining FVIII:C above 1\% will not protect all patients from bleeding. ${ }^{7,8}$ Evaluation of the association between FVIII:C and bleeding pattern is important and can support evidence-based tailoring of prophylaxis independently of the factor concentrate used.

We have used data from the guardian clinical trial program to examine bleeding risks according to FVIII:C with a recombinant FVIII (rFVIII) molecule during prophylaxis in patients with severe hemophilia A. The product, turoctocog alfa (NovoEight ${ }^{\circledR}$, Novo Nordisk Health Care AG, Zürich, Switzerland), is a B-domain-truncated rFVIII. Data from three studies were combined: a pivotal trial in adults and adolescents (guardian 1; NCT00840086), ${ }^{9}$ a pivotal trial in children (guardian 3; NCT01138501), ${ }^{10}$ and an extension trial (guardian 2, which was an extension trial of guardian 1 and 3; NCT00984126). ${ }^{11}$ Estimated mean (95\% confidence interval) spontaneous $\mathrm{ABR}$ in these studies were 4.32 (3.34-5.59) (pivotal trial in adults and adolescents), 1.69 (0.94-3.03) (pivotal trial in children), and 1.34 (1.07-1.68) (extension trial)..$^{9-11}$

The PK characteristics of turoctocog alfa, which have been extensively studied using standard PK assessments (based on plasma FVIII:C) and a population PK model, have been shown to be consistent over time, reproducible between lots and similar to those of other commercially available FVIII products. ${ }^{12}$

Defining the relationship between FVIII:C and bleeding pattern is important not only because bleeding frequency is an important outcome, but also to guide robust, individualized prophylactic treatment schedules. Given the importance of spontaneous bleeding in hemophilia, we assessed the relationship between FVIII:C and ABR, as a measure of bleeding frequency for spontaneous bleeds, including spontaneous joint bleeds, which were recorded in dosing diaries by patients with severe hemophilia A who received prophylaxis with turoctocog alfa in the guardian clinical trials.

\section{Methods}

\section{Trial design and patients' eligibility}

The pivotal trials (guardian 1 and 3) were multinational, phase III trials assessing prophylaxis with turoctocog alfa in adults/adolescents ( $\geq 12$ years) and pediatric patients (0-11 years), respectively. All patients had severe hemophilia A (FVIII $\leq 1 \%$ ) without inhibitors and had already been exposed to FVIII for $\geq 150$ days (adults/adolescents) or $\geq 50$ days (children). ${ }^{9,10}$ Patients completing the pivotal trials or phase I PK trials could continue into the open-label extension trial (guardian 2). ${ }^{13}$ Patients received turoctocog alfa as prophylaxis (20-50 IU/kg every second day or 20-60 IU/kg three times weekly, depending on age), as well as for treatment of bleeds. All three trials are now complete. Patients in the trials reported their dosing and bleeding data in diaries. Baseline joint disease at pivotal study entry was not assessed; joint disease in the current analysis was therefore inferred based on treatment history.

\section{Pharmacokinetic assessment in the pivotal studies}

Consistent with the International Society of Thrombosis and Haemostasis FVIII product guidelines ${ }^{14}$ the pivotal trials included a single-dose PK assessment in a subset of patients after the first dose and in adults/adolescents after 3-6 months' treatment; this involved nine (adults/adolescents) or six (children) sampling time points up to $48 \mathrm{~h}$ after injection of the product., ${ }^{9,10,12}$ In addition, samples were taken from all patients before and after dosing at the routine visits. All plasma samples were analyzed at a central laboratory using a one-stage clot assay. ${ }^{12,15}$ and post-dose FVIII:C values were entered into the PK model.

\section{Population pharmacokinetic modeling}

Population PK modeling was conducted with the nonlinear, mixed-effects modeling software NONMEM (v.7.1.2, ICON Development Solutions, Ellicott City, MD, USA). Variability between patients was quantified within the population PK mode $1^{12}$ by including between-patient variability on PK parameters using a log-normal distribution with no correlation. Allometric scaling based on body weight was applied to clearance $(\mathrm{CL})$ and volume of distribution $(\mathrm{Vd})$ parameters, and age was estimated as a linear covariate on CL. A combined proportional and additive residual error model was used.

\section{Exposure-response analysis: estimating the association between factor VIII activity and annualized bleeding rate}

The exposure-response analysis of the association between predicted FVIII:C and spontaneous bleeds, including joint bleeds, was conducted with the statistical software R3.2.3 (Comprehensive R Archive Network [CRAN] project, University of Münster, Germany).

The population PK model estimates were applied to diaryrecorded dosing from all patients receiving prophylaxis with turoctocog alfa in the pivotal and extension trials who had appropriate records, regardless of whether patients reported a spontaneous bleed or not, to produce a predicted FVIII:C time course for each patient over the entire span of prophylaxis for that patient. These predicted profiles had peaks following each recorded dose and subsequent gradual decline, taking into account the contributions from the last three doses at any time point (Figure 1). Each patient's time on prophylaxis was divided into five clinically meaningful categories of predicted FVIII:C: $0-1 \%,>1-5 \%$, $>5-15 \%,>15-50 \%$, and $>50 \%$.

Subsequently, the diary-reported bleeding (spontaneous, including joint bleeds) data were compared to the predicted FVIII:C-time-profiles to estimate the FVIII:C-bleeding pattern relationships. Patients were excluded from the analysis if they switched from prophylaxis to on-demand treatment during the extension trial, received only on-demand treatment throughout the extension trial, had missing diary returns, or failed to document time of bleeding for $>50 \%$ of recorded bleeds.

For the purposes of this analysis, we have described bleeds as those bleeds that patients reported in their diary returns. This is to acknowledge that the bleeds were subjectively evaluated by the patient.

For each patient, exposure time to turoctocog alfa, mean FVIII:C, and number of spontaneous bleeds (including joint bleeds) requiring treatment (based on the patient's diary entries and excluding re-bleeds within $48 \mathrm{~h}$ ) were calculated for each predicted FVIII:C category. To assess the association between FVIII:C and ABR, bleeding data were used to estimate ABR for 
each of the five predicted FVIII:C categories for each patient, using negative binomial regression and predictions of a parametric model. Relationships between ABR and mean FVIII:C were evaluated for the overall dataset (pivotal and extension trials), and by trial phase (pivotal vs. extension) and patients' age (adults/adolescents [ $\geq 12$ years] vs. children [0-11 years]). The proportion of time in which patients were predicted to achieve a FVIII:C level was calculated by taking the patient-years of exposure (PYE) for a FVIII:C category range, dividing it by the overall PYE, and multiplying it by $100 \%$.

The risk of bleeding episodes and FVIII:C levels was also analyzed using survival analysis. For each patient and trial phase, the time on a given factor level to the first bleed was calculated. The time to first bleed is presented in Kaplan-Meier plots displaying the proportion of patients without a bleed at a given time. For each trial phase, the difference between the FVIII:C levels was tested using a log-rank test for statistical significance.

\section{Results}

\section{Patients and exposure to turoctocog alfa}

The demographics of the PK subgroups in the pivotal trials $(n=50)$ and of the patients participating in these trials and from whom post-dose FVIII:C measurements were used in the analysis are shown in Table 1 . The analysis population, which was limited to patients receiving prophylaxis, comprised 232 patients across the pivotal and extension trials. One patient was excluded from the analysis as over $50 \%$ of bleeds recorded by this patient lacked a time recording, leaving 231 patients (63 children, 168 adults). Total PYE for adults/adolescents and children in the pivotal trials were 77 and 23, respectively, and 498 and 217, respectively, in the extension trial.

\section{Population pharmacokinetic modeling}

The total pool of PK data in the population PK model comprised the PK profiles from the PK subgroups in the pivotal trials supplemented with post-dose measurements from routine clinical visits for patients participating in these trials $(n=231)$. The PK of turoctocog alfa was found to align well with a one-compartmental model (i.e., the elimination of turoctocog alfa after a single intravenous bolus dose was approximately log-linear) as described by the equation:

$$
C_{p}(t)=\frac{D}{V} \cdot e^{-\frac{C L}{V} \cdot t}=\frac{D}{V} \cdot e^{-k t}
$$

where $D$ is dosage, $t$ is the time since dose, $V$ is the volume of distribution, $C L$ is clearance, $k$ is the elimination rate constant, and $\mathrm{e}$ is the base of the natural logarithm ( 2.72). One-compartmental behavior is indicative of the compound being distributed mainly within the bloodstream itself. The population PK parameter estimates are shown in Table 2. ${ }^{12}$ Therefore, the one-compartmental model was selected as the basis for the population PK analysis, using turoctocog alfa data derived from the one-stage clot assay for all patients. ${ }^{12}$

The volume of distribution for an individual of weight $W, V(W)$, was calculated according to the equation below, where $W_{\text {ref }}$ represents the body weight of the reference individual $(70 \mathrm{~kg})$ :

$$
V(W)=\left(\frac{W}{W_{\text {ref }}}\right)^{e_{V}}
$$

Clearance for an individual with a weight $W$ and age $A$ (CL/ $W$, A)), was calculated according to the formula below, where $A_{\text {ref }}$ represents the age of the reference individual age (20 years):

$$
C L(W, A)=\left(\frac{W}{W_{\text {ref }}}\right){ }^{e_{C L}} \cdot\left(1+k_{\text {age }}\left(A-A_{\text {ref }}\right)\right)
$$

\section{Predicted factor VIII activity - bleeding relationship}

Overall, there were 1,237 spontaneous bleeds (324 during the pivotal phase and 913 during the extension phase), of which 1,063 occurred in adults/adolescents and 174 in children (Table $3)$. The vast majority ( $n=1,055 ; 85.29 \%$ ) of spontaneous bleeds were joint bleeds; more adults/adolescents than children suffered joint bleeds. The proportion of time at FVIII:C levels $>1 \%$ and the PYE that were used to calculate this parameter across patient populations and trial phases are shown in Table 3. It was predicted that overall FVIII:C levels $\geq 1 \%$ were achieved for $85.64 \%$ of the time.

Mean spontaneous bleeds, including joint bleeds, decreased as FVIII:C increased (Tables 4 and 5), indicating an exposure-bleeding relationship. This relationship was evident in both the pivotal and extension trial phases for both types of bleed. However, for each FVIII:C activity category, lower ABR were observed in the extension phase than in the pivotal phase. A FVIII:C-bleeding relationship was also apparent for the two age groups of adults/adolescents and children, although children had a lower ABR than adults/adolescents within each FVIII:C activity category (Table 5).

At low FVIII:C, ABR were lower during the extension phase than in the pivotal phase for both adults/adolescents and children (Figure 2). The difference in ABR between adults/adolescents and children was particularly evident for the pivotal phase; during the extension phase, the ABR for each age group was similar for spontaneous bleeds, with slightly lower ABR in children compared with adults/adolescents. Similar relationships between FVIII:C and ABR were evident for joint bleeds (Online Supplementary Figure S1).

Using Kaplan-Meier plots, analyzed over a fixed period of time (60 days), the time to first spontaneous bleeding was longer during times at higher FVIII:C levels (Figure 3). A similar pattern was apparent with joint bleeds (Online Supplementary Figure S2). Hence, patients were more protected during times at higher FVIII:C levels, particularly during the extension phase. In general, patients remained bleed-free for longer at any level of FVIII:C in the extension phase, particularly at the lower FVIII:C levels. Log-rank calculations showed a significant difference between each FVIII:C category in the proportion of patients without a bleed during the pivotal phase $(P<0.0001)$ and the extension phase $(P<0.0001)$.

\begin{tabular}{|c|c|c|c|c|}
\hline \multirow[b]{2}{*}{ Parameters } & \multicolumn{2}{|c|}{ Adults/adolescents } & \multicolumn{2}{|c|}{ Children } \\
\hline & $\begin{array}{c}\text { PK } \\
\text { sulggroup }\end{array}$ & All* & $\begin{array}{c}\text { PK } \\
\text { sulpgroup }\end{array}$ & All* \\
\hline Number of patients & 22 & 168 & 28 & 63 \\
\hline $\begin{array}{l}\text { Age on entering } \\
\text { program, years; } \\
\text { mean (SD) }\end{array}$ & $\begin{array}{l}24.00 \\
(7.88)\end{array}$ & $\begin{array}{c}28.98 \\
(12.15)\end{array}$ & $\begin{array}{c}5.96 \\
(2.76)\end{array}$ & $\begin{array}{c}6.08 \\
(2.91)\end{array}$ \\
\hline $\begin{array}{l}\text { Body weight, } \\
\text { kg; mean (SD) }\end{array}$ & $\begin{array}{c}71.84 \\
(12.44) \\
\end{array}$ & $\begin{array}{c}73.5 \\
(18.13) \\
\end{array}$ & $\begin{array}{c}24.43 \\
(10.50) \\
\end{array}$ & $\begin{array}{c}24.6 \\
(10.03)\end{array}$ \\
\hline
\end{tabular}

Table 1. Demographics of patients included in the exposure-response analysis.

*Adults/adolescents and children from the pivotal trials or patients who completed the phase I pharmacokinetic trials could enter the extension trial. Patients were excluded from the exposure-response analysis if they switched from prophylaxis to on-demand treatment during the extension trial, received only on-demand treatment throughout the extension trial, had missing diary returns, or failed to document time of bleeding for $>50 \%$ of recorded bleeds. PK: pharmacokinetic;SD: standard deviation. 


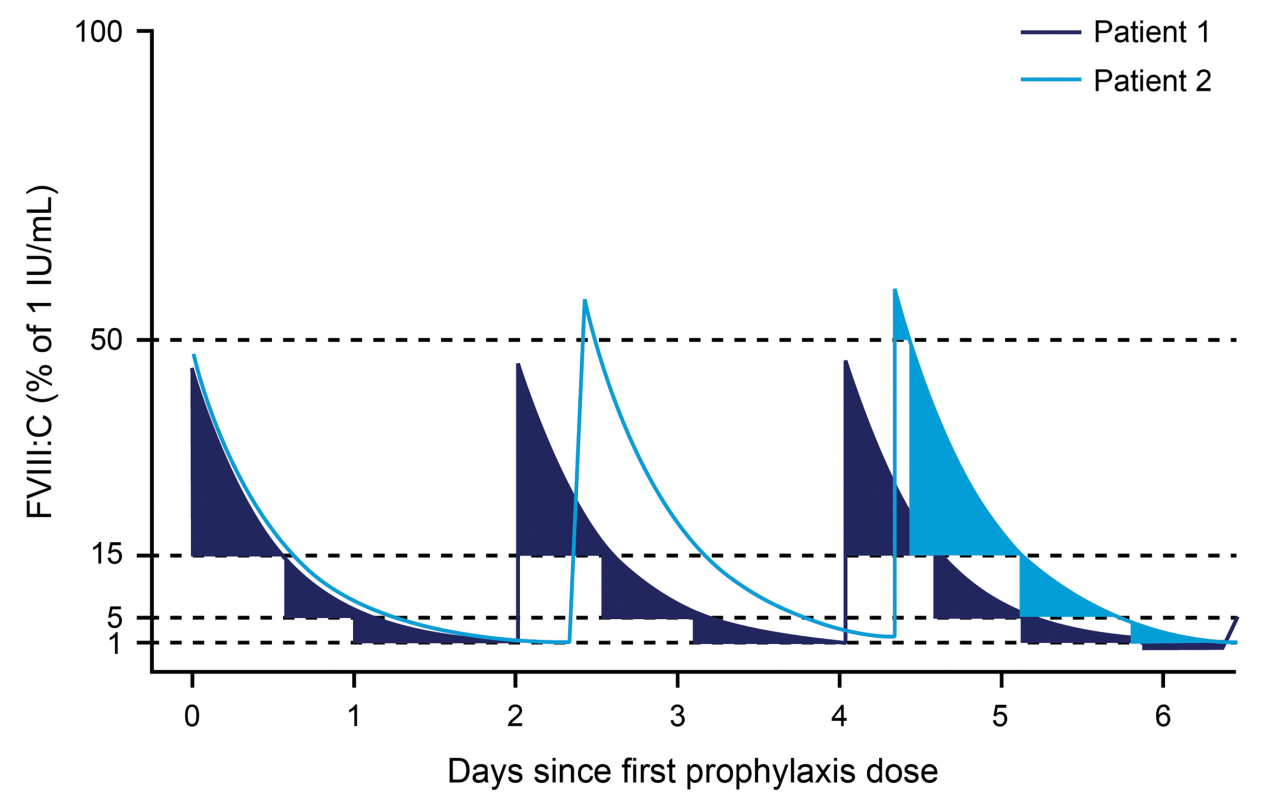

Figure 1. Patients' time spent in different FVIII:C activity ranges predicted from dosing diaries. The first week of prophylaxis is illustrated for two representative patients on turoctocog alfa dosed three times weekly. Predicted profiles of FVIII:C activity were calculated from the reported patients' diary information on doses and timing of doses. Both patients exhibited a dosing interval pattern of 2-2-3 days in their first week, although the last two doses were delayed and higher for patient 2 than for patient 1. Triangles represent the area under the curve (AUC) of FVIII:C contributing to the mean FVIII:C value for each range. The horizontal span of each triangle defines the time spent in the relevant FVIII:C range. Both the $A U C$ and time spans were calculated across dosing intervals and patient years. Actual trough FVIII:C was measured inconsistently and locally, and was not generally used to guide treatment decisions.

\section{Discussion}

We used a population PK model and diary-recorded dosing data to estimate FVIII levels in patients with severe hemophilia A undergoing prophylaxis with turoctocog alfa and assessed the relationship between FVIII:C and ABR using diary-recorded data on bleeds. This approach confirmed an association between higher FVIII activity levels and reduced risk of spontaneous bleeding, apparent across all ages and trial phases. Bleeding rates were lower in the extension phase than in the pivotal phase at the same FVIII:C levels, consistent with previous spontaneous ABR data from these studies. ${ }^{13}$ The FVIII exposure-bleeding relationship was also apparent for both adults/adolescents and children. This effect, however, disappeared during the extension phase; after a period of 6 months of prophylaxis during the pivotal studies, the bleeding risk in adult patients approached that of the pediatric population. This suggests that patients' characteristics at baseline accounted for the difference in bleeding risk between the age groups. The use of a large number of data on outcomes and their potential modifiers, combined from patients in different age groups and trial phases, is a strength of our study. It is reasonable to assume that the observed relationship between FVIII:C and ABR, as well as the role of modifying factors, is largely independent of the factor concentrate used in the source studies. The main conclusions conveyed by our work are therefore for prophylaxis with any factor concentrate in severe hemophilia A.

Although our analysis showed that ABR decreased with higher FVIII:C, it was not possible to identify a single FVIII:C level that would prevent bleeding in all patients. For example, an ABR of $<2$ was achieved when FVIII:C was $>15 \%$ in the pivotal phase but had already been achieved when FVIII was $>1 \%$ in the extension phase. We would speculate that, over time, the patients' clinical condition (including joint disease activity) improved and the bleeding risk decreased. In addition, the effect of previous on-demand treatment on ABR would have diminished over time and may have contributed to the lower bleeding
Table 2. Population pharmacokinetic parameters.

\begin{tabular}{lc} 
Parameter & $\begin{array}{c}\text { Population parameter } \\
\text { estimate (CV) }\end{array}$ \\
$\mathrm{CL}_{70 \mathrm{~kg}, 20 \mathrm{y}}(\mathrm{mL} / \mathrm{h})^{\mathrm{a}}$ & $302(0.32)$ \\
$\mathrm{V}_{70 \mathrm{~kg}}(\mathrm{~L})^{\mathrm{b}}$ & $3.46(0.22)$ \\
Allometric scaling exponent for body weight & 0.95 \\
on clearance $\left({ }^{e} \mathrm{CL}\right)$ & \\
Allometric scaling exponent for body weight on $\mathrm{V}\left({ }^{e} \mathrm{~V}\right)$ & 0.86 \\
\hline Age effect on $\mathrm{CL}(1 /$ year $)$ & -0.01 \\
\hline
\end{tabular}

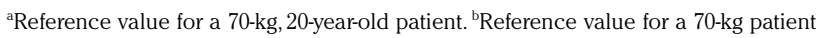
$\mathrm{CL}$ : clearance; CV: inter-individual variability; V: volume of distribution.

rates in the extension phase. Over a third of adult/adolescent patients received only on-demand treatment prior to entering the pivotal trial, ${ }^{9}$ potentially affecting joint disease and bleeding risk at study entry, even at higher FVIII:C levels. In children (who commonly start prophylactic regimens early on), an $A B R<2$ was achieved at any FVIII:C level $\geq 1 \%$, consistent with the notion that pristine joints have a lower risk of bleeding during prophylaxis. The lower bleeding rates in adults in the extension phase compared with the pivotal phase could therefore indicate a "calming-down" effect of prophylaxis on joint disease activity.

These results support the notion that treatment outcomes (and study endpoints) such as ABR would not only be influenced by FVIII exposure, but also by patient-related factors such as age, activity level, or joint status. Different hemophilia products, including extended halflife factor concentrates, can transform FVIII exposure, but direct comparison of outcomes across studies may be confounded by such patient-related factors. Similar confounders may have to be considered when comparing results of non-factor replacement therapy or even gene therapy to results achieved with FVIII replacement.

Our findings on the FVIII exposure-response relationship support and build on data from other modeling analyses using negative binomial distribution in patients with hemophilia A. Collins et al. ${ }^{6}$ established the importance of 
FVIII trough levels and protection from bleeding in patients with severe hemophilia A. Valentino et al. ${ }^{16}$ linked higher peak FVIII levels, higher area under the curve and time with FVIII $>20$ IU/dL ( $>20 \%$ ) with increased protection against joint and non-joint bleeding. den Uijl et al. suggested that joint bleeds decreased to approximately zero at FVIII levels $>12 \%$ in patients with mild to severe hemophilia A. As such, it would be expected that FVIII trough levels of $15 \%$ would prevent most bleeds, considering that patients with a FVIII trough of $15 \%$ are likely to

Table 3. Time spent at each FVIII:C range and bleeding characteristics after turoctocog alfa prophylaxis.

\begin{tabular}{|c|c|c|c|c|c|}
\hline \multirow[t]{2}{*}{ Parameters } & \multicolumn{2}{|c|}{ Pivotal } & \multicolumn{2}{|c|}{ Extension } & \multirow[t]{2}{*}{ Overall } \\
\hline & Adults/adolescents & Children & Adults/adolescents & Children & \\
\hline PYE & 77 & 23 & 498 & 217 & 815 \\
\hline Total numbers of spontaneous bleeds & 287 & 37 & 776 & 137 & 1237 \\
\hline Total numbers of spontaneous joint bleeds & 251 & 24 & 684 & 96 & 1055 \\
\hline \multicolumn{6}{|c|}{ Proportion of time (\%) in each FVIII activity range } \\
\hline $0-1 \%$ & 15.58 & 21.74 & 12.85 & 16.60 & 14.36 \\
\hline$>1-5 \%$ & 27.27 & 26.09 & 26.10 & 26.73 & 26.38 \\
\hline$>5-15 \%$ & 25.97 & 21.74 & 25.50 & 20.73 & 24.17 \\
\hline$>15-50 \%$ & 28.57 & 21.74 & 29.32 & 23.04 & 27.48 \\
\hline$>50 \%$ & 3.90 & 8.70 & 6.22 & 12.90 & 7.85 \\
\hline
\end{tabular}

FVIII: factor VIII; FVIII:C: factor VIII activity; PYE: patient-years of exposure.

Table 4. FVIII:C ranges and annualized bleeding rate of spontaneous bleeds and spontaneous joint bleeds.

\begin{tabular}{|c|c|c|c|c|c|}
\hline FVIII activity range & $\begin{array}{l}\text { Patient } \\
\text { years of } \\
\text { exposure }\end{array}$ & $\begin{array}{l}\text { Total number } \\
\text { of spontancous } \\
\text { bleeds }\end{array}$ & $\begin{array}{l}\text { Total number } \\
\text { of spontaneous } \\
\text { joint bleeds }\end{array}$ & $\begin{array}{l}\text { Overall mean } \\
\text { spontaneous ABR } \\
\text { (negative binomial } \\
\text { estimate, } 95 \% \mathrm{Cl} \text { ) }\end{array}$ & $\begin{array}{c}\text { Overall mean } \\
\text { spontaneous } \\
\text { joint ABR } \\
\text { (negative binomial } \\
\text { estimate, } 95 \% \text { CI) }\end{array}$ \\
\hline $0-1 \%$ & 116.6 & 303 & 241 & $\begin{array}{c}4.16 \\
(3.23-5.40)\end{array}$ & $\begin{array}{c}3.44 \\
(2.56-4.66)\end{array}$ \\
\hline$>1-5 \%$ & 214.5 & 396 & 335 & $\begin{array}{c}2.65 \\
(2.12-3.34)\end{array}$ & $\begin{array}{c}2.28 \\
(1.77-2.96)\end{array}$ \\
\hline$>5-15 \%$ & 197.1 & 371 & 337 & $\begin{array}{c}2.14 \\
(1.69-2.74)\end{array}$ & $\begin{array}{c}1.99 \\
(1.53-2.60)\end{array}$ \\
\hline$>15-50 \%$ & 223.7 & 154 & 133 & $\begin{array}{c}0.76 \\
(0.58-0.99)\end{array}$ & $\begin{array}{c}0.67 \\
(0.50-0.90)\end{array}$ \\
\hline$>50 \%$ & 63.6 & 13 & 9 & $\begin{array}{c}0.21 \\
(0.11-0.36)\end{array}$ & $\begin{array}{c}0.15 \\
(0.07-0.31)\end{array}$ \\
\hline
\end{tabular}

ABR: annualized bleeding rate; 95\% CI: 95\% confidence interval; FVIII: factor VIII; FVIII:C: FVIII activity.

Table 5. FVIII:C ranges and annualized bleeding rate of spontaneous bleeds by subgroup.

\begin{tabular}{|c|c|c|c|c|c|c|c|c|}
\hline \multirow[t]{3}{*}{ FVIII activity range } & \multicolumn{4}{|c|}{$\begin{array}{c}\text { Mean spontancous ABR } \\
\text { (negative binomial estimate, } 95 \% \text { Cl) } \\
\text { Analyses population }\end{array}$} & \multicolumn{4}{|c|}{$\begin{array}{c}\text { Mean spontancous joint ABR } \\
\text { (negative binomial estimate, } 95 \% \text { CI) } \\
\text { Analyses population }\end{array}$} \\
\hline & \multicolumn{2}{|c|}{ By trial phase } & \multicolumn{2}{|c|}{ By age group } & \multicolumn{2}{|c|}{ By trial phase } & \multicolumn{2}{|c|}{ By age group } \\
\hline & Pivotal & Extension & $\begin{array}{l}\text { Adults/ } \\
\text { adolescents }\end{array}$ & Children & Pivotal & Extension & $\begin{array}{c}\text { Adults/ } \\
\text { adolescents }\end{array}$ & Children \\
\hline \multirow[t]{2}{*}{$0-1 \%$} & 7.16 & 2.15 & 4.86 & 2.68 & 5.94 & 1.75 & 4.17 & 1.83 \\
\hline & $(5.15-10.02)$ & $(1.58-2.97)$ & $(3.64-6.52)$ & $(1.61-4.60)$ & $(4.01-8.94)$ & $(1.23-2.56)$ & $(3.02-5.83)$ & $(0.95-3.73)$ \\
\hline \multirow[t]{2}{*}{$>1-5 \%$} & 4.15 & 1.69 & 3.29 & 0.95 & 3.55 & 1.44 & 2.88 & 0.67 \\
\hline & $(3.02-5.76)$ & $(1.30-2.23)$ & (2.61-4.17) & $(0.49-1.90)$ & $(2.46-5.22)$ & $(1.07-1.98)$ & $(2.22-3.78)$ & $(0.32-1.49)$ \\
\hline \multirow[t]{2}{*}{$>5-15 \%$} & 2.95 & 1.64 & 2.64 & 0.71 & 2.76 & 1.49 & 2.48 & 0.58 \\
\hline & $(2.06-4.25)$ & $(1.23-2.23)$ & $(2.04-3.44)$ & $(0.40-1.27)$ & $(1.87-4.10)$ & $(1.09-2.08)$ & $(1.89-3.28)$ & $(0.27-1.29)$ \\
\hline \multirow[t]{2}{*}{$>15-50 \%$} & 0.99 & 0.66 & 0.92 & 0.32 & 0.92 & 0.56 & 0.82 & 0.23 \\
\hline & $(0.60-1.61)$ & $(0.49-0.89)$ & $(0.69-1.22)$ & $(0.16-0.64)$ & $(0.55-1.52)$ & $(0.40-0.79)$ & $(0.60-1.13)$ & $(0.10-0.55)$ \\
\hline \multirow[t]{2}{*}{$>50 \%$} & 0.47 & 0.19 & 0.35 & 0.03 & 0.23 & 0.14 & 0.26 & 0.00 \\
\hline & $(0.08-1.44)$ & $(0.09-0.34)$ & $(0.19-0.59)$ & $(0.00-0.15)$ & $(0.01-1.02)$ & $(0.06-0.31)$ & $(0.12-0.50)$ & $(0.00-0.00)$ \\
\hline
\end{tabular}

ABR: annualized bleeding rate; $95 \%$ CI: $95 \%$ confidence interval; FVIII: factor VIII; FVIII:C: FVIII activity. 
have a higher FVIII:C for the majority of time between doses. When we describe FVIII:C at $15 \%$ in our analysis, this reflects a momentary FVIII:C value between peaks and troughs, rather than a trough value (Figure 1). As such, bleeding risk will not be the same in a patient at a momentary FVIII:C level of $15 \%$, if the trough in this patient is much lower, compared with a patient with a trough level of $15 \%$, who would have much higher FVIII:C for most of the time.

In addition to reporting negative binomial distribution estimates of ABR, we used Kaplan-Meier analysis and logrank statistics to assess the bleeding risk at different FVIII levels. This analysis provides a slightly different perspective on the data by depicting the proportion of bleed-free patients over a given period of observation, confirming the impact of FVIII levels on the risk of bleeding. It should be noted, however, that there was no plateau reached during the analysis period of 2 months in our study, and it can be expected that the proportion of bleed-free patients would decrease further over time. The proportion of bleed-free patients depicted in Figure 3 should not, therefore, be compared directly to proportions of bleed-free patients reported from other studies over different periods of time.

Our analysis also has some potential sources of error such as: the misinterpretation of symptoms of pain as a bleed; delayed recognition of bleeds resulting in a shift of the start of a bleed to some time after the next injection; and inaccuracy of injection records. Assuming these potential errors decreased during the guardian trials, this may provide some explanation for the decline in ABR over time. On the other hand, we cannot exclude underreporting of bleeds by patients during the extension phase, particularly when visits changed from every 2 months during the pivotal phase, to every 6 months during the extension phase.

PK data from patients treated with FVIII products can be described using one- or two-compartmental PK models. ${ }^{17}$ Two-compartmental models may not be identifiable from PK data with less intensive sampling, such as data from children. ${ }^{17}$ Furthermore, one-compartmental models are less sensitive than two-compartmental models to the handling of observations below "limits of quantification". This is because a smaller number of parameters are to be estimated from the given pool of data. While there is no well-established consensus regarding handling in population PK modeling, ${ }^{18}$ these choices can have profound effects for FVIII PK modeling. ${ }^{19}$

Overall, our data suggest that trough level targets need to be adapted according to a patient's age and previous treatment and should be revised regularly over time. We hypothesize that higher levels may be required in patients
A

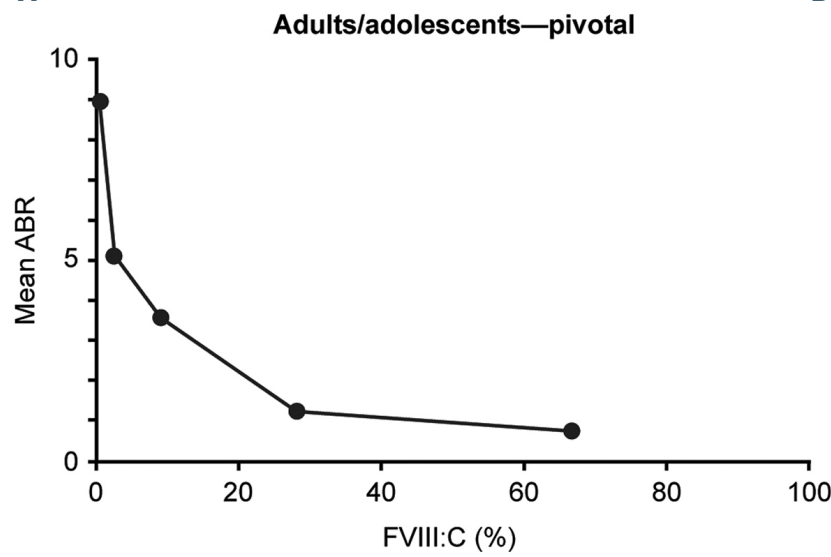

C

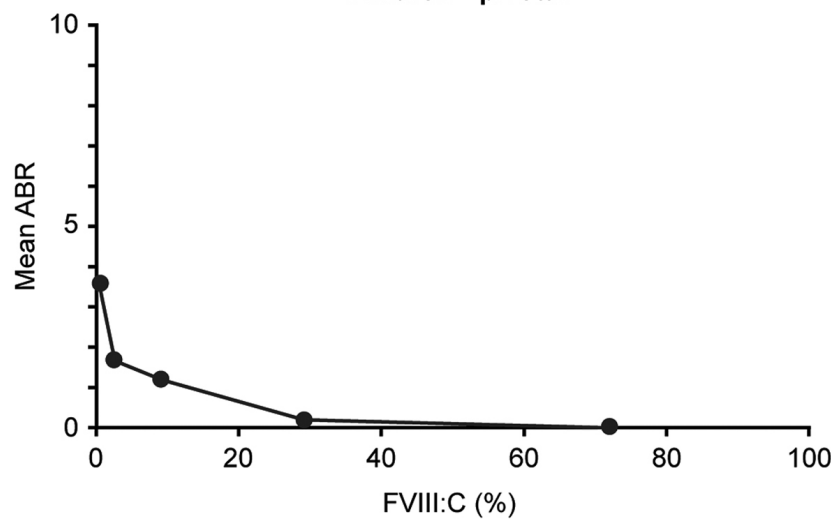

B

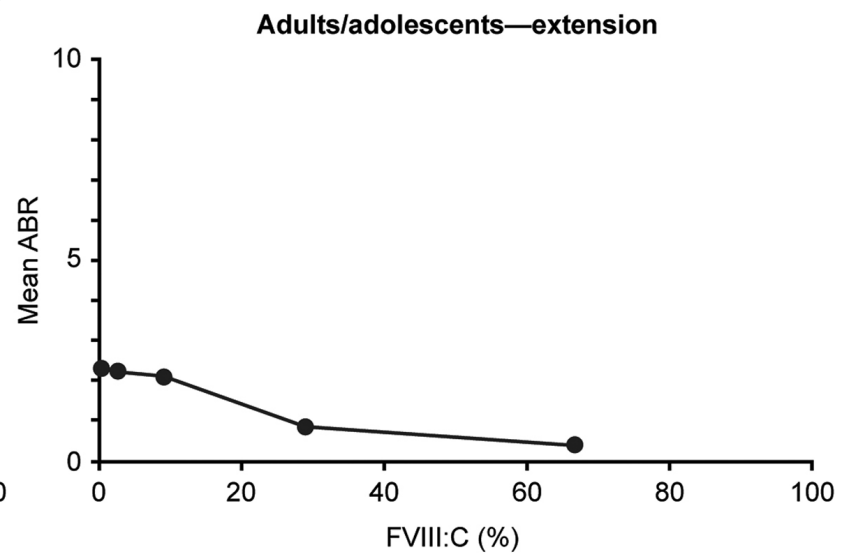

D

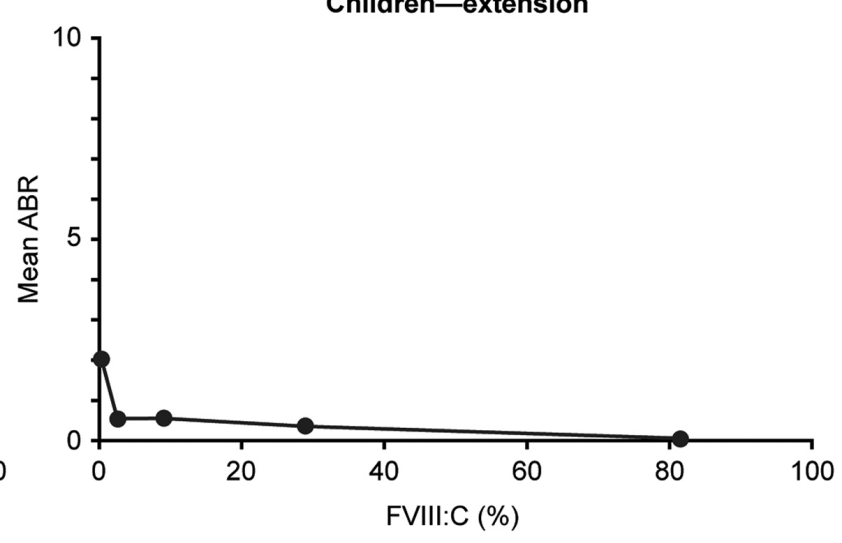

Figure 2. Estimates of annualized bleeding rates for spontaneous bleeds for five FVIII:C categories in the two trial phases and age groups. (A-D) Negative binomial estimates of annualized bleeding rate (ABR) for five FVIII:C categories (0-1\%, >1-5\%, >5-20\%, >20-50\%, and >50\%) for spontaneous bleeds during the pivotal (A) and extension (B) phases for adults/adolescents, and during the pivotal (C) and extension (D) phases for children. FVIII:C: factor VIII activity. 


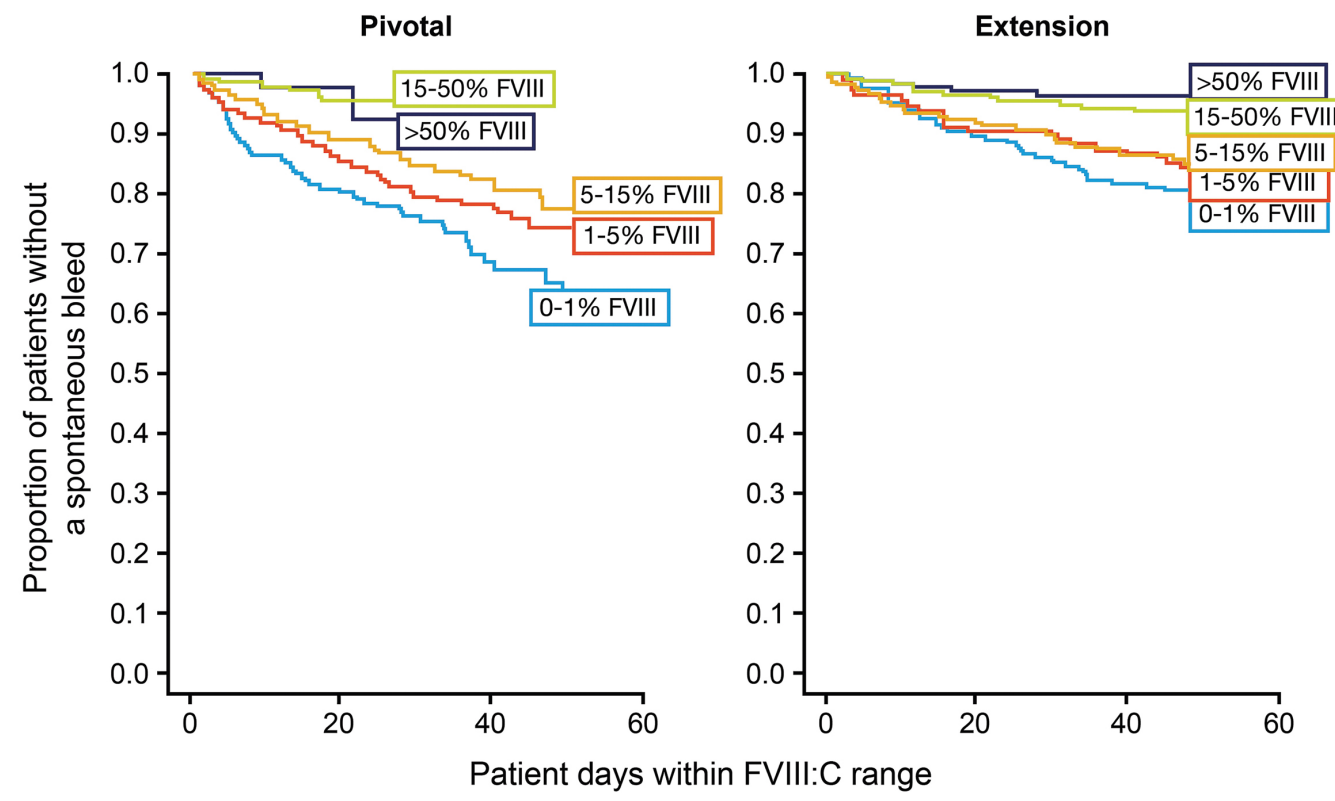

Figure 3. Kaplan-Meier estimates of the proportion of patients without a spontaneous bleed according to predicted FVIII:C during the pivotal and extension trial phases. FVIII:C: FVIII factor VIII activity.

with active joint disease (particularly target joints and synovitis), and the intensity of prophylaxis may be reduced in those without active joint disease who have not bled for some time. Further prospective studies are needed to evaluate this approach.

Bleeds are currently used as the main endpoint for studies in hemophilia, both for newly developed factor concentrates, as well as for non-factor replacement products. Characteristic features of novel factor concentrates include, but are not limited to, longer half-life and improved preservation of post-translational modification. It could be tempting to compare $\mathrm{ABR}$ across studies of these products, but it should be kept in mind that ABR is a multifactorial outcome. Our analysis illustrates that ABR changes over time and depends on patients' characteristics.

In conclusion, in patients of all ages with severe hemophilia A who were treated with turoctocog alfa, ABR for spontaneous bleeds, including joint bleeds, decreased as FVIII:C increased, indicating an exposure-response relationship. The data presented in the current study suggest that patient-related factors and treatment history influence the FVIII:C level needed to protect patients from bleeding. ABR reported in clinical trials not only reflect efficacy of the FVIII product, but also the characteristics of the population under study. ${ }^{7,20}$ The choice of prophylactic regimens to target certain trough levels should take into account the patient's age, joint disease activity, and other determinants of bleeding risk.

\section{Disclosures}

AT has received research support, honoraria, or consultation fees from Alnylam, Bayer, Biogen Idec, Biotest, Bristol-Myers Squibb, Boehringer Ingelheim, CSL Behring, Leo Pharma, Novo Nordisk, Octapharma, Pfizer, Roche, Shire, and SOBI. VJ-Y has received reimbursement for attending symposialcongresses and/or honoraria for speaking and/or for consulting, and/or funds for research from Takeda, Bayer, CSL-Behring, Grifols, Novo Nordisk, Sobi, Roche, Octapharma and Pfizer. RK has received support, honoraria, or consultation fees from Bayer, Biogen Idec, Biotest, Bristol-Myers Squibb, Boehringer Ingelheim, CSL Behring, Grifols, Leo Pharma, Novo Nordisk, Octapharma, Pfizer, Roche, Shire, and SOBI. TS has received consultation and speaker fees from Bayer, Bioverativ, Chugai, CSL Behring, IL Japan, JB Pharma, Kaketsuken, Merck Sharp \& Dohme, Nihon-Pharma, Novo Nordisk, Pfizer, Sekisui Medical, and Shire. AG is an employee of Novo Nordisk A/S. ES has attended and received funds for Advisory Boards from Bayer, Grifols, Kedrion, Novo Nordisk, Octapharma, Pfizer, Roche, Shire, and SOBI. She attended Speaker Bureaus for, and received funds from, Bayer, Bioverativ, CSL Behring, Grifols, Kedrion, Novo Nordisk, Octapharma, Pfizer, Roche, Shire, and SOBI. FAK and SL have no conflicts of interest to disclose.

\section{Contributions}

$A G$, representing Novo Nordisk $A / S$, provided the statistical analyses of the data; AT contributed to the study design and interpreted the results; all authors critically wrote or revised the intellectual content of the manuscript, reviewed and/or commented on each draft, and approved the final version for submission.

\section{Acknowledgments}

These trials were sponsored by Novo Nordisk A/S (Bagsvoerd, Denmark). The authors thank the patients with severe hemophilia $A$ and their families/caregivers, as well as the investigators, pharmacists, nurses, and trial staff at each center for participating in these trials. The authors would also like to thank Brigitte Brand-Staufer (Novo Nordisk Health Care AG) and Lars Korsholm for their scientific advice and critical review of the manuscript. Medical writing support was provided by Jo Fetterman, PhD (Parexel, UK).

\section{Funding}

This work was funded by Novo Nordisk A/S (Bagsvoerd, Denmark). Novo Nordisk's policy on data sharing may be found at https://novonordisk-ctts.app-trialscope.com/how-access-clinical-trial-datasets. 


\section{References}

1. Biggs R, MacFarlane RG. Haemophilia and related conditions: a survey of 187 cases. $\mathrm{Br}$ J Haematol. 1958;4(1):1-27.

2. White GC $2^{\text {nd }}$, Rosendaal F, Aledort LM, Lusher JM, Rothschild C, Ingerslev J. Definitions in hemophilia. Recommendation of the scientific subcommittee on factor VIII and factor IX of the scientific and standardization committee of the International Society on Thrombosis and Haemostasis. Thromb Haemost. 2001; 85(3):560.

3. Nilsson IM, Hedner U, Ahlberg A. Haemophilia prophylaxis in Sweden. Acta Paediatr Scand. 1976;65(2):129-135.

4. Manco-Johnson MJ, Abshire TC, Shapiro $\mathrm{AD}$, et al. Prophylaxis versus episodic treatment to prevent joint disease in boys with severe hemophilia. N Engl J Med. 2007; 357(6):535-544.

5. Srivastava A, Brewer AK, MauserBunschoten EP, et al. Guidelines for the management of hemophilia. Haemophilia. 2013;19(1):e1-47.

6. Collins PW, Blanchette VS, Fischer K, et al. Break-through bleeding in relation to predicted factor VIII levels in patients receiving prophylactic treatment for severe hemophilia A. J Thromb Haemost. 2009; 7(3):413-420

7. den Uijl IE, Mauser Bunschoten EP, Roosendaal G, et al. Clinical severity of haemophilia A: does the classification of the 1950s still stand? Haemophilia. 2011; 17(6):849-853.

8. den Uijl IE, Fischer K, Van Der Bom JG, Grobbee DE, Rosendaal FR, Plug I. Analysis of low frequency bleeding data: the associ- ation of joint bleeds according to baseline FVIII activity levels. Haemophilia. 2011; 17(1):41-44.

9. Lentz SR, Misgav M, Ozelo $M$, et al. Results from a large multinational clinical trial (guardian ${ }^{\mathrm{TM}}$ 1) using prophylactic treatment with turoctocog alfa in adolescent and adult patients with severe haemophilia A: safety and efficacy. Haemophilia. 2013;19(5):691-697.

10. Kulkarni R, Karim FA, Glamocanin S, et al Results from a large multinational clinical trial (guardian ${ }^{\mathrm{TM}} 3$ ) using prophylactic treatment with turoctocog alfa in paediatric patients with severe haemophilia A: safety, efficacy and pharmacokinetics. Haemophilia. 2013;19(5):698-705.

11. Lentz SR, Janic D, Kavakli K, et al. Longterm safety and efficacy of turoctocog alfa in prophylaxis and treatment of bleeding episodes in severe haemophilia A: final results from the guardian 2 extension trial. Haemophilia. 2018;24(6):e391-e394.

12. Jimenez-Yuste V, Lejniece $S$, Klamroth R, et al. The pharmacokinetics of a B-domain truncated recombinant factor VIII, turoctocog alfa (NovoEight ${ }^{\mathbb{Q}}$ ), in patients with hemophilia A. J Thromb Haemost. 2015; 13(3):370-379

13. Ozelo M, Misgav M, Abdul KF, et al. Longterm patterns of safety and efficacy of bleeding prophylaxis with turoctocog alfa (NovoEight ${ }^{\circledR}$ ) in previously treated patients with severe haemophilia A: interim results of the guardian ${ }^{\mathrm{TM}} 2$ extension trial. Haemophilia. 2015;21(5):e436-e439.

14. Lee M, Morfini M, Schulman S, and the Factor VIII/Factor IX Scientific and Standardization Committee of the International Society for Thrombosis and Haemostasis. Scientific and Standardization
Committee Communication. The design and analysis of pharmacokinetic studies of coagulation factors. https://www.isth.org/. Last update: 21 March 2001. Accessed in September, 2017.

15. Bolton-Maggs PH, Perry DJ, Chalmers EA, et al. The rare coagulation disordersreview with guidelines for management from the United Kingdom Haemophilia Centre Doctors' Organisation. Haemophilia. 2004:10(5):593-628.

16. Valentino LA, Pipe SW, Collins PW, et al. Association of peak factor VIII levels and area under the curve with bleeding in patients with haemophilia A on every third day pharmacokinetic-guided prophylaxis. Haemophilia. 2016;22(4):514-520.

17. Bjorkman S, Oh M, Spotts G, et al Population pharmacokinetics of recombinant factor VIII: the relationships of pharmacokinetics to age and body weight Blood. 2012;119(2):612-618.

18. Ahn JE, Karlsson MO, Dunne A, Ludden TM. Likelihood based approaches to handling data below the quantification limit using NONMEM VI. J Pharmacokinet Pharmacodyn. 2008;35(4):401-421.

19. Garmann D, McLeay S, Shah A, Vis P, Maas Enriquez M, Ploeger BA. Population pharmacokinetic characterization of BAY 81 8973, a full-length recombinant factor VIII lessons learned - importance of including samples with factor VIII levels below the quantitation limit. Haemophilia. 2017; 23(4):528-537.

20. Fischer K, Chowdary P, Collins P, et al. Modelling FVIII levels for predictions of zero spontaneous-joint bleeding in a cohort of severe hemophilia A subjects with target joints initiated on tertiary prophylaxis. Blood. 2016;128(22):2576. 